\section{The role of the dental surgeon in detecting osteoporosis: the OSTEODENT study}

\author{
H. Devlin, ${ }^{1}$ P. Allen, ${ }^{2}$ J. Graham, ${ }^{3}$ R. Jacobs, ${ }_{1}^{4}$ K. Nicopoulou-Karayianni, ${ }_{1}^{5}$ \\ C. Lindh, ${ }^{6}$ E. Marjanovic, ${ }^{7}$ J. Adams, ${ }^{8}$ S. Pavitt, ${ }^{9}$ P. van der Stelt ${ }^{10}$ \\ and K. Horner ${ }^{11}$
}

IN BRIEF

- Women at high risk of osteoporosis can be identified by dentists using information from panoramic radiographs supplemented by a few clinical questions.

- Dentists may contribute to a woman's general health by facilitating onward referral to medical colleagues.

- The software that carries out the radiographic assessment requires minimal dentist input to work optimally.

\begin{abstract}
Objective To determine if thinning ( $<3 \mathrm{~mm}$ width) of the lower cortical border of the mandible on dental panoramic radiographs, as well as other clinical risk factors, may provide a useful diagnostic test for osteoporosis in young postmenopausal women. Design Six hundred and fifty-two subjects (age range 45-70 years) were involved in this multi-centre, cross-sectional study. Setting Patients were recruited from centres in Leuven (Belgium), Athens (Greece), Manchester (UK), and Malmo (Sweden). Subjects and methods The subject's age, body weight, whether the patient took hormone replacement therapy or had a history of low trauma fracture were used to form a clinical osteoporosis risk assessment (the OSteoporosis Index of RISk or OSIRIS index). Each patient also received a dental panoramic radiographic examination. Results One hundred and forty subjects had osteoporosis involving at least one of the measurement sites (lumbar spine, femoral neck or total hip). Those with osteoporosis tended to have a low OSIRIS score and a thinned cortical mandibular border. The area under the ROC curve for using both cortical width and OSIRIS to predict osteoporosis was $0.90(95 \% \mathrm{Cl}=$ 0.87 to 0.92 ). There was a significant improvement in the diagnostic ability of the combined OSIRIS and cortical width test over both tests applied separately $(p<0.001)$. The cost effectiveness of the cortical width and OSIRIS model was improved by using a high specificity threshold rather than high sensitivity. However, this analysis ignores the costs associated with missed cases of osteoporosis. Conclusion Dentists have a role to play in the detection and referral of patients at high risk of osteoporosis.
\end{abstract}

\section{INTRODUCTION}

Osteoporosis is a serious disease, but treatment can be instituted when early detection is made possible. Hip fractures, in particular, are associated with significant mortality and morbidity in the elderly, ${ }^{1}$ but in one study, less than one fifth (18\%) of high risk people had received medical treatment for osteoporosis before the occurrence of hip fracture. ${ }^{2}$ The current failure to assess and treat patients at high risk of osteoporosis may be partly due to

'School of Dentistry, University of Manchester; ${ }^{2,3,7,8} \mathrm{Im}$ aging Science and Biomedical Engineering, University of Manchester: ${ }^{4}$ Oral Imaging Centre, Katholieke Universiteit, Belgium; ${ }^{5}$ Oral Diagnosis and Radiology, Dental School of Athens; ${ }^{6}$ Faculty of Odontology, Malmo University, Sweden; 9,115chool of Dentistry, University of Manchester: ${ }^{10} \mathrm{ACTA}$, The Netherlands

${ }^{*}$ Correspondence to: Dr Hugh Devlin

Email:hugh.devlin@manchester.ac.uk

\section{Online article number E16}

Refereed Paper - accepted 14 November 2008

DOI: $10.1038 /$ sj.bdj.2008.317

${ }^{\circledR}$ British Dental Journal 2008; 204: E16 insufficient resources or time, but a failure of health professionals to identify risk factors and refer the patient for definitive diagnosis using dual-energy $\mathrm{X}$-ray absorptiometry (DXA) is an important contributory factor.

In an attempt to improve this situation, several clinical risk 'tests' have been developed as a means of identifying subjects who would benefit from further investigation. The contribution of clinical risk factors (such as OSIRIS) to the primary prevention of osteoporotic fractures is at present under consideration by the World Health Organisation and the National Institute for Health and Clinical Excellence (NICE). OSIRIS is a weighted combination of those clinical risk factors that are known to independently predict whether a patient has osteoporosis. These indices, however, have not gained universal acceptance as a routine diagnostic test because of their poor specificity in detecting patients at increased risk of osteoporosis. ${ }^{3,4}$
Harrison and Adams ${ }^{5}$ found that clinical risk indices misclassified unacceptably large numbers of osteoporotic women, with consequent decreased cost effectiveness. Recently, we reported the use of mandibular cortical width measurements on dental panoramic radiographs (DPRs) as an alternative method of identifying patients with osteoporosis., ${ }^{6,7}$ The underlying rationale for this is the enormous number of DPRs taken in dental practice. We propose a strategy of the dentist referring individuals with a thin mandibular cortex and other clinical risk factors for further DXA investigation. Detection of a thinned cortex on DPRs using specially developed computer software ${ }^{8}$ has been found to be a good predictor of systemic osteoporosis, and because the method is automatic it is also convenient.

This study has two aims. The first was to determine the diagnostic efficacy of combining the OSIRIS clinical index with the cortical width measurement 
on radiographs, using multivariate statistical analysis. The second aim was to evaluate the cost effectiveness of using this combined test as a basis for further referral for central dual energy X-ray absorptiometry (DXA), using the 90\% sensitivity and 90\% specificity values as thresholds.

\section{METHODS}

This work forms part of the OSTEODENT study, a collaborative project funded by the European Commission Fifth Framework Programme 'Quality of Life and Management of Living Resources'. The methodology of subject recruitment and examination has been fully described previously, ${ }^{7}$ and is summarised here. With Ethics Committee approval, female subjects (aged 45-70 years) were recruited consecutively into the study following their informed consent. Subjects were recruited from each centre using publicity material and by word-of-mouth, but this patient group may not be representative of a primary dental care population. The study included all female volunteers and patients in this age group, with subjects excluded only if they suspected that they might be pregnant. All subjects were interviewed and provided information about their age, weight, medication and fracture history. Patients were recruited from centres in Leuven (Belgium), Athens (Greece), Manchester (UK), and Malmo (Sweden). Six hundred and seventy-one subjects were recruited into the study. The bone mineral density of the total hip could not be measured in two subjects and a further eight subjects were found to be aged less than 45 years, so their data were not included in any further analysis. A further nine radiographs were either lost, digitally corrupted or of poor diagnostic quality. The remaining 652 subjects formed the study population and underwent a clinical risk assessment of osteoporosis using the OSIRIS questionnaire (OSteoporosis Index of RISk) and computer cortical width measurement. One hundred and forty subjects had osteoporosis involving at least one measurement site.

\section{Dental radiographs}

Each subject underwent a dental panoramic radiographic examination while biting on a spherical, steel ball bearing

Table 1 Cortical width and OSIRIS data from 652 subjects, 140 of whom were osteoporotic at one of the measurement sites

\begin{tabular}{l|l|l|l|l} 
& \multicolumn{2}{|l|}{ Osteoporotic $(n=140)$} & \multicolumn{2}{l}{ Normal $(n=512)$} \\
\hline & OSIRIS & Cortical width & OSIRIS & Cortical width \\
\hline Mean (SD) & $-0.24(2.5)$ & $3.0(0.6)$ & $3.40(2.9)$ & $3.7(0.6)$ \\
\hline Maximum & 6.6 & 5.1 & 14 & 5.8 \\
\hline Minimum & -5.9 & 1.8 & -4.6 & 2.2
\end{tabular}

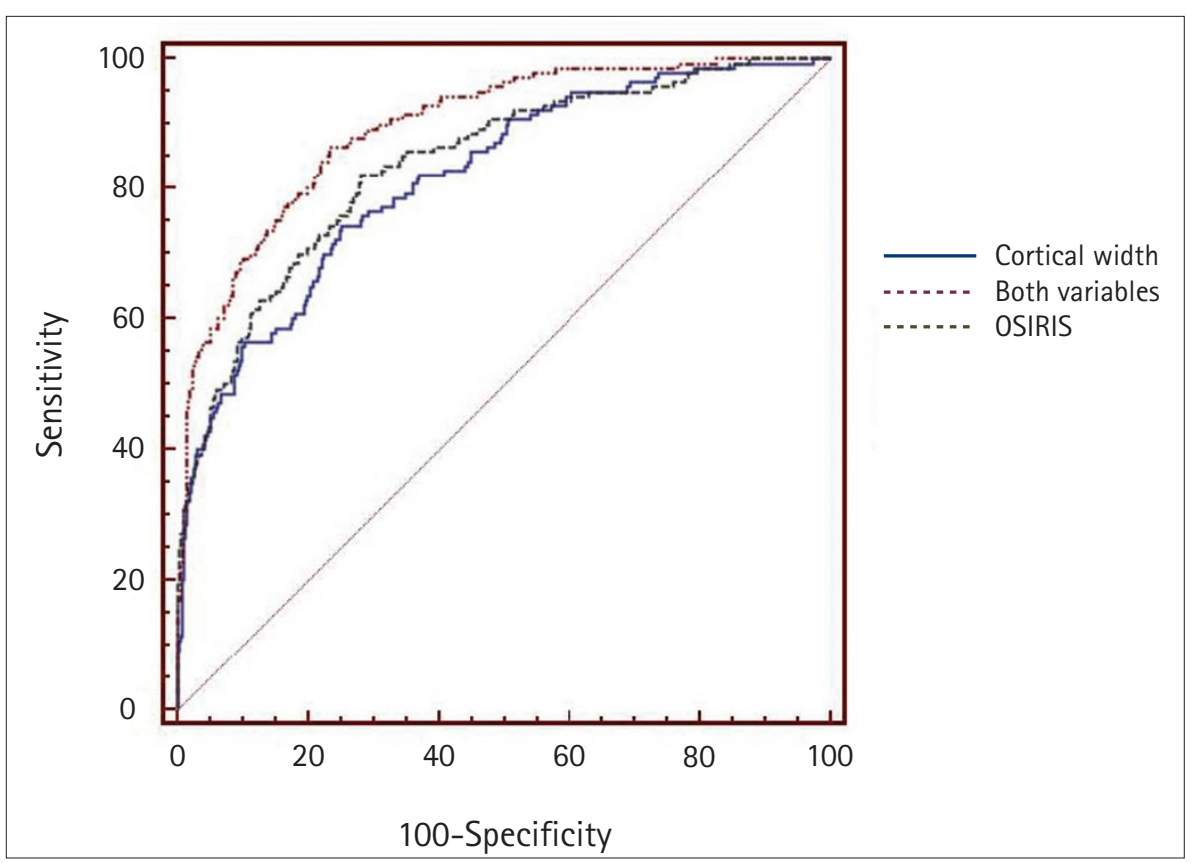

Fig. 1 ROC curve of OSIRIS, cortical width measurements on radiograph, and the effect of combining both variables

(3.175 $\mathrm{mm}$ diameter), used to calculate the image magnification. The Leuven (Belgium) and Malmo (Sweden) centres used a Cranex III (Soredex, IL, USA) dental panoramic radiography machine whereas Athens (Greece) and Manchester (UK) used a Planmeca (Planmeca USA, Roselle, IL, USA). In Leuven, a photostimulable phosphor plate system for image capture and digital read out was used, but other centres used a conventional film/cassette. Typical imaging parameters for panoramic radiography were $70 \mathrm{kV}$ at $8 \mathrm{~mA}$ for $15 \mathrm{~s}$. All of the radiographs were digitised using a Kodak LS85 digitiser (Eastman Kodak, Rochester, NY) at a resolution of 25.64 pixels/mm.

The mandibular cortex was automatically detected on the digitised panoramic radiographs using software based on Active Shape Model search ${ }^{9}$, which is a sophisticated computer imaging technique. Its width was measured by the method described by Allen et al. ${ }^{8}$

\section{DXA Examination}

Central DXA of the proximal femur and lumbar spine was performed at each of the four centres. The World Health Organisation (WHO) criteria were used to diagnose osteoporosis, ie using DXA to identify those with a bone mineral density T-score value 2.5 SD or more below the mean value of the young sex matched reference population at any of the lumbar spine, femoral neck or total hip measurement sites. This was used as a 'goldstandard' measure of osteoporosis.

\section{Osteoporosis Index of Risk (OSIRIS)}

The Osteoporosis Index of Risk (OSIRIS) is based on four variables: ${ }^{10}$ age, body weight, current hormone replacement therapy (HRT) use and history of previous low impact fracture. The index is calculated by adding together:

- Age multiplied by -2 (rounded down to the nearest integer)

- Weight in kg multiplied by 2 (rounded down to the nearest integer) 
- +2 if a current user of HRT

- -2 if a history of low trauma fracture.

An OSIRIS score of lower than -3 indicates a high risk of low BMD, between +1 and -3 an intermediate risk and greater than +1.0 a low risk. ${ }^{11}$

\section{Statistical analysis}

Student's t-test was used to analyse the significance of the differences between OSIRIS and cortical width values in the osteoporotic and normal individuals.

Discriminant analysis, using the variables cortical width and OSIRIS score, was used to derive the probability of osteoporosis in an individual subject. The model was evaluated using a leaveone-out cross validation strategy to avoid biasing the estimates of discrimination ability. Models were calculated from the entire data set except one, which was used as the test datum. This process was repeated using each of the patients in turn as a test datum. The calculated probability of osteoporosis from each of the experiments was used to generate an ROC curve. The resulting area under the ROC curve was compared with that of OSIRIS and the cortical width measurements. The 90\% sensitivity and 90\% specificity thresholds were used to calculate the numbers of subjects correctly and incorrectly classified as osteoporotic.

\section{Cost effectiveness}

Two strategies were compared to determine those who should receive further investigation using dual energy X-ray absorptiometry. Criteria values for the combined cortical width and clinical analysis used either (a) 90\% sensitivity or (b) $90 \%$ specificity. In calculating the cost per patient correctly diagnosed with osteoporosis, the costs used for central DXA were $£ 50$ per patient and for the OSIRIS index were $£ 5$ per patient. Both of these costs estimates have been used recently in other publications by our research group. ${ }^{5}$

\section{RESULTS}

The mean difference in mandibular cortical width between osteoporotic and healthy patients $(0.718 \mathrm{~mm})$ was highly significant $(\mathrm{t}=12.83, \mathrm{p}<0.0001$ ) (Table 1). The difference between osteoporotic

Table 2 The numbers (and \% of the total sample) referred for DXA using a threshold of $90 \%$ sensitivity. This guaranteed that the majority of patients with osteoporosis would be referred for further DXA examination, but 164 (56.6\%) of the referred patients would have a normal BMD. In total, 178 (or $27.3 \%$ ) of the 652 patients were misclassified

\begin{tabular}{|l|l|l|l} 
& No referral for DXA & Refer for DXA & Total \\
\hline \multirow{3}{*}{ Osteoporosis present } & 14 & 126 & \multirow{2}{*}{140} \\
\cline { 2 - 4 } & $2.10 \%$ & $19.30 \%$ & \\
\hline \multirow{2}{*}{ Osteoporosis absent } & 348 & 164 & \multirow{2}{*}{512} \\
\cline { 2 - 4 } & $53.40 \%$ & $25.20 \%$ & \multirow{2}{*}{652} \\
\hline \multirow{2}{*}{ Total } & 362 & 290 &
\end{tabular}

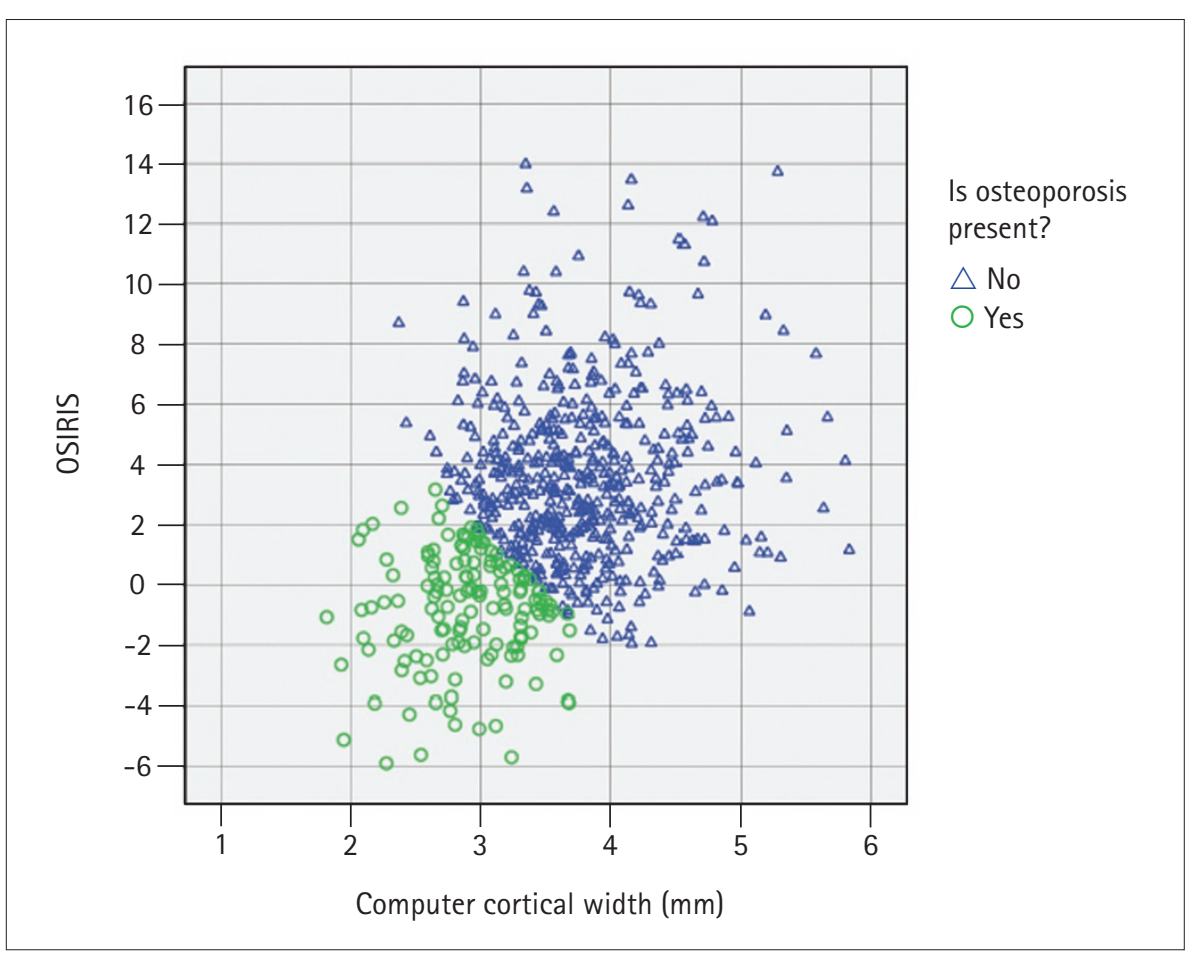

Fig. 2 The variables OSIRIS and cortical width are plotted. The $90 \%$ specificity value has been used as a threshold and those who are or are not indicated for referral for central DXA are indicated (ie predicted probability of osteoporosis of either less than or greater than $34.5 \%$ )

and normal patients' OSIRIS indices (3.62) was also highly significant $(\mathrm{t}=$ 13.54, $\mathrm{p}<0.0001)$.

Discriminant analysis was used to obtain a linear combination of weighted average of cortical width and OSIRIS variables that resulted in the best separation between those with and without osteoporosis in our sample. The resulting discriminant score was used to distinguish between the two groups. Wilk's lamba (the ratio of the withingroup sum of squares to the total sum of squares) was $0.683\left(\chi^{2}=247.6, \mathrm{df}=2, \mathrm{p}\right.$ $<0.001$ ). Therefore the two groups (those with and without osteoporosis) differed in their mean discriminant score, and $79 \%$ of cases were correctly assigned to the groups. Both cortical width and OSIRIS variables contributed equally to the prediction of group membership because they had similar standardised regression coefficients (OSIRIS $=0.682$, cortical width $=0.634$ ).

A model derived from a sample will usually fit it better than another sample obtained from the same population. In further leave-one-out cross validation analysis, each case was classified using all the other data to derive the function except that one. A similar but less biased estimate of the correct classification rate of $78.8 \%$ of cross-validated grouped cases was obtained.

Using this model, the correlations between the probability of osteoporosis 
and the BMD at the lumbar spine, femoral neck and total hip were $-0.60,-0.64$ and -0.61 , respectively. These correlations were highly significant, $\mathrm{p}<0.01$.

The area under the ROC curve (Fig. 1) recorded separately for OSIRIS was $0.84(95 \% \mathrm{CI}=0.81$ to 0.87$)$ and for the cortical width was 0.82 (95\% CI 0.79 to 0.85). The difference between both ROC curve areas was 0.021 , which was not significantly different $(95 \% \mathrm{CI}=-0.022$ to 0.064$), p=0.335$. The area under the ROC curve for the predicted probability produced by the linear discriminant analysis in the cross validation experiment (cortical width and OSIRIS) was 0.90 ( $95 \% \mathrm{CI}=0.87$ to 0.92 ). There was a significant improvement in the diagnostic ability of the combined OSIRIS and cortical width test over both tests applied separately ( $\mathrm{p}<0.001)$.

Using the combined OSIRIS and cortical width data, an 'Osteodent Index' was calculated giving the risk of osteoporosis. An operating point on the ROC curve with a specificity value of 90\% (95\% CI $=87.1$ to 92.5 ) and corresponding value of sensitivity of $69 \%$ (95\% CI $=60.2$ to 76.1) was selected. By using a high specificity value, at the expense of sensitivity, the minimum number of patients would be sent for unnecessary further investigations. Using this criterion value gave a test with a positive likelihood ratio of 6.9 and negative likelihood ratio of 0.35 . The diagnostic odds ratio, the ratio of positive likelihood ratio divided by negative likelihood ratio, was 19.7.

The OSIRIS values were plotted against cortical width for the sample of 652 patients. Using the 90\% specificity threshold for the combined variable, the sample was divided into those predicted as being at either high or low risk of osteoporosis. Figure 2 shows the scatterplot of OSIRIS index and cortical width, with assignment to either high or low risk of osteoporosis. Our previous work $^{12}$ has shown that the optimal decision boundary of whether to further refer patients lies at a $3 \mathrm{~mm}$ cortical width. Figure 2 shows that in a patient with a $3 \mathrm{~mm}$ cortical width, only when the OSIRIS value is greater than 1.83 is referral not indicated.

There is some overlap of osteoporotic and non-osteoporotic OSIRIS and cortical

Table 3 The numbers (and \% of the total sample) referred for DXA using a threshold of $\mathbf{9 0} \%$ specificity. Only $\mathbf{5 0}$ (or $10 \%$ ) of patients with normal BMD would be referred for further DXA examination. In total, 94 (or 14.4\%) of the 652 subjects were misclassified

\begin{tabular}{l|l|l|l} 
& No referral for DXA & Refer for DXA & Total \\
\hline \multirow{3}{*}{ Osteoporosis present } & 44 & 96 & \multirow{2}{*}{140} \\
\cline { 2 - 4 } & $6.70 \%$ & $14.70 \%$ & \multirow{2}{*}{512} \\
\hline \multirow{2}{*}{ Osteoporosis absent } & 462 & 50 & \multirow{2}{*}{652} \\
\cline { 2 - 4 } & $70.90 \%$ & $7.70 \%$ & \\
\hline \multirow{2}{*}{ Total } & 506 & 146 &
\end{tabular}

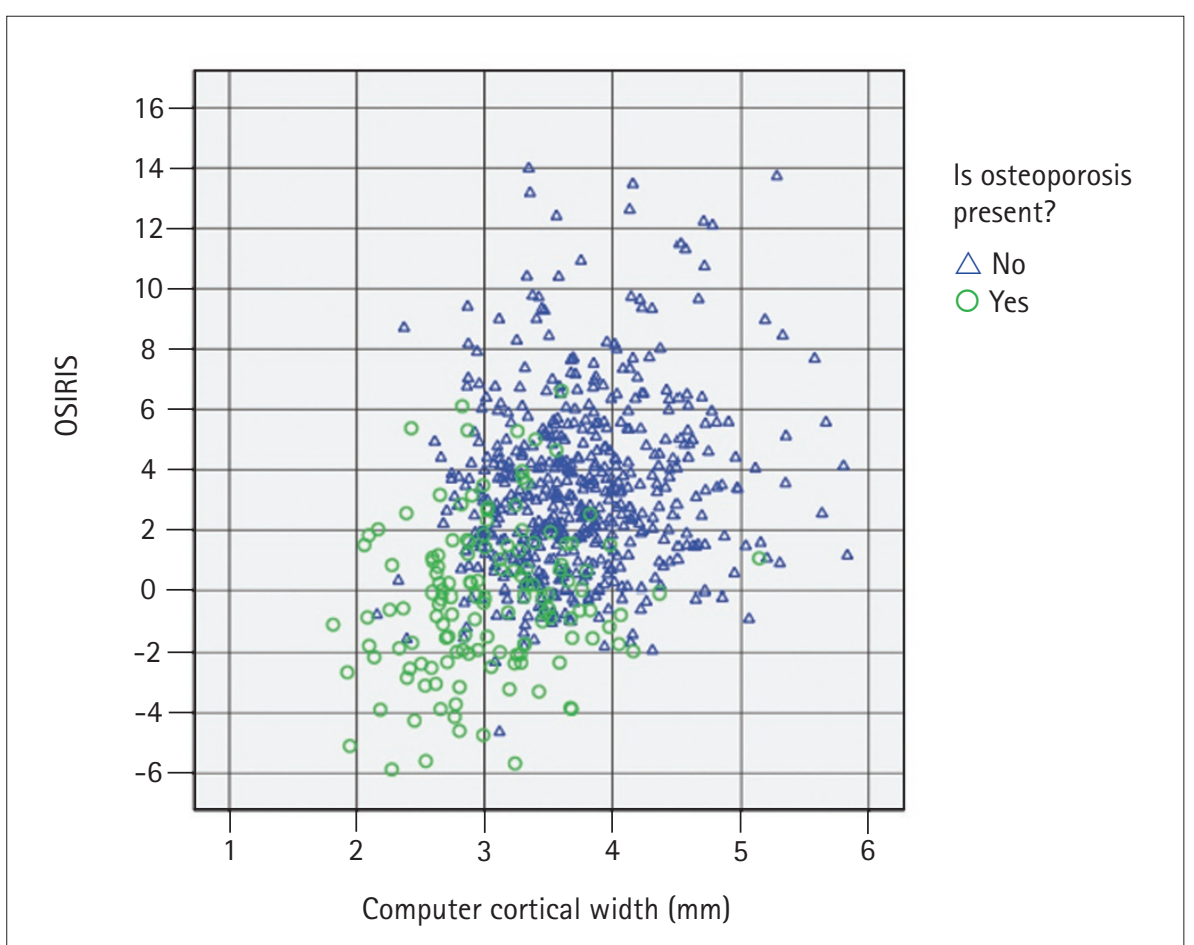

Fig. 3 Cortical width measurements plotted against OSIRIS, with each point represented as either osteoporotic or normal according to DXA. While osteoporotic subjects tend to group towards low values of both parameters, there is extensive overlap between the two groups

width values (Fig. 3). Those patients with osteoporosis tend to be grouped towards the lower values of both parameters.

Table 2 shows the false positive and false negative assignments arising from a referral decision at $90 \%$ sensitivity for the combined cortical width and OSIRIS data. The corresponding specificity was $68 \%$ and the diagnostic odds ratio was 18.73. While using a high sensitivity ensures that only $10 \%$ of osteoporotic patients would fail to be referred for further investigation, 56.6\% of those referred would have a normal BMD. The cost of this strategy was $£ 141$ per osteoporotic patient diagnosed.

An alternative decision strategy is to adopt a high specificity operating point (Table 3). Using a threshold of $90 \%$ specificity, results in a referral of only 50 $(10 \%)$ of those with a normal BMD, but with the disadvantage that 44 out of the 140 osteoporotic patients (31.4\%) would be missed. The cost of this strategy was $£ 110$ per osteoporotic patient diagnosed.

\section{DISCUSSION}

In this study, we have described a casefinding strategy, where a combination of a clinical index (OSIRIS) and automatically measured width of mandibular cortex, is a technique with good diagnostic accuracy in predicting low bone mineral density at the hip or spine. The diagnostic odds ratio, the ratio of positive likelihood ratio divided by negative likelihood ratio, measures the performance of a test and a value above 20 
indicates a diagnostic test with strong evidence for efficacy. ${ }^{13}$ Our test, providing a diagnostic odds ratio of 19.7, falls into this category. Furthermore, the fact that the combined test performed better than either the clinical or the radiological test alone demonstrates that they are not providing the same information, but rather complementary information. It is therefore worthy of further clinical trial. Other case-finding strategies that have combined the information from clinical risk factors and selective use of BMD have also proven to be more successful in identifying high-risk patient groups. ${ }^{14}$ Clinical risk factors have been shown to independently predict hip fracture risk, ${ }^{15}$ but the balance between a simply administered assessment is often at odds with the requirement for a comprehensive assessment of all possible risk factors. Therefore, we plan to further assess the ability of our diagnostic strategy to predict hip fracture in our target population of young post-menopausal women.

In the United States and other industrialised nations, patients are increasingly aware of the benefits of disease prevention, as well as the long-term cost saving with healthcare. ${ }^{16}$ What then could be the clinical role for this form of testing for low bone density? Our detection strategy would be used to select those who would undergo further DXA, a case finding approach that follows the UK Royal College of Physicians Guidelines. ${ }^{17}$ Millions of dental radiographs are taken by dentists annually, with dental radiographs accounting for nearly 25\% of all medical radiographic exposures. ${ }^{18}$ Using dentists to select postmenopausal women at high risk of osteoporosis has the advantage that patients are seen regularly from this age group, and that there is an increasing use of radiographs by dentists for diagnosis. ${ }^{19}$

The diagnostic efficacy of the radiographic test alone makes it clearly unsuitable as a screening test, because it performs no better than the simple clinical risk assessment; it also has greater cost and an associated X-ray exposure. We have previously suggested that dentists should refer patients for DXA opportunistically using DPRs that they have taken for the usual dental purposes. Would the combined test be justifiably used as a screening test, at least in the age group of women examined in this study? The decision partly depends on the cost effectiveness of this strategy.

With wide scale dental radiographic and clinical identification of osteoporotic patients, increased healthcare costs in the short-term would be inevitable because of increased demand for DXA services. Europe is already underresourced for central DXA machines. ${ }^{20}$ Our study cannot predict the numbers of femoral neck fractures prevented if our methodology was introduced, but age and a history of previous fracture (which contribute to the OSIRIS index) and a low femoral neck BMD are clinical risk factors which play a role in femoral fracture risk. ${ }^{21,22}$

In the UK, the National Osteoporosis Society has recommended that postmenopausal women given peripheral $\mathrm{X}$-ray absorptiometry be classified into three risk categories. ${ }^{23}$ In the first group at high risk of osteoporosis, treatment is recommended, particularly if accompanied by other risk factors. In the second group, the patients are referred for central DXA for further confirmation, and in the third group no additional action is recommended. In this context, we developed an analogous strategy using the combined cortical width and OSIRIS variables to categorise patients into three groups of differing osteoporosis risk. Two thresholds were chosen based on the 90\% sensitivity (the low threshold) and the 90\% specificity values (the high threshold), as described by Harrison and Adams. ${ }^{5}$ The 90\% sensitivity value was the predicted probability value of osteoporosis represented by the $10^{\text {th }}$ percentile and the $90 \%$ specificity value was the predicted value of nonosteoporosis represented by the $90^{\text {th }}$ percentile. However, the value of this whole approach is limited by the medical side effects and the high cost of providing long-term drug therapy for those in the highest risk group when they do not have osteoporosis.

We therefore compared using either threshold values of $90 \%$ sensitivity or $90 \%$ specificity for the combined cortical width and OSIRIS variable as two strategies of referral for DXA for those patients considered 'at risk' of osteoporosis. Using a test with 90\% specificity (£110 per diagnosed osteoporotic patient) provided a more cost effective option than using 90\% sensitivity (£141 per osteoporotic patient). This is due to the large number of patients with normal bone mineral density that were referred unnecessarily for DXA, and therefore the comparatively low yield of osteoporotic patients. There was a cost of $£ 233$ per diagnosed osteoporotic patient if all patients in the study received central DXA. This analysis ignores the costs associated with the undiagnosed osteoporotic patients as these were difficult to define in this study. The authors hope that some of these individuals would be identified through further opportunistic testing using either our methodology on a future occasion or other techniques, such as dual energy X-ray absorptiometry or quantitative ultrasound. We also hope to use our test methodology and any subsequent patient treatment to examine the incremental cost-effectiveness ratio per quality adjusted life year. In addition, if the net benefit of our methodology is to be assessed, the distribution of risk assessment cost over the population must be calculated.

Case-finding strategies are prone to operator variability and error. For example, evidence from chest radiography taken in an emergency hospital department has shown that only $25 \%$ of patients with radiologically evident vertebral fractures received a diagnosis of osteoporosis or any treatment. ${ }^{24}$ Our own research using observer measurements of cortical width has demonstrated that the weakness lies in observer variability. One can postulate that dentists in a primary care setting would be inaccurate in making some measurements by hand. ${ }^{7,25}$ Our methodology involves a computer-measured mandibular cortical width to make the initial diagnosis of a high risk of osteoporosis, and following consultation with the patient, the dentist can either provide follow-up clinical questions, such as an OSIRIS clinical risk index, or refer to a specialist.

The cost effectiveness of our proposed methodology is dependent on the prevalence of osteoporosis in our study population $(21.5 \%)$ being comparable to that 
of the UK population. In a study based in Hull, UK, the prevalence of osteoporosis of the hip and spine in general practice was similar (24\%), but their study population consisted of women in their seventh decade. ${ }^{26}$ The mean age of our study sample was 55 years ( $s d=6.1$ ), and the age range (45-70 years) was chosen to test an asymptomatic population which was more representative of young postmenopausal women attending a general dental practice. Our osteoporotic screening method could be made more cost effective if restricted to an elderly population; fewer misdiagnoses are then likely because the incidence of vertebral and hip fractures increases exponentially with age. ${ }^{27}$

Poor radiographic technique is common in general dental practice and may limit the usefulness of our technique. For example in a study by Rushton et $a l .,{ }^{28}$ the image of the lower border of the mandible was at least partially absent in 9\% of panoramic radiographs. Using digital panoramic radiographs will approximately halve the reject rate of films as about half of faults are due to chemical processing of film. ${ }^{28}$ Patient positioning faults could be reduced by using better positioning aids, further training of dentists with an emphasis on quality assurance, and using only suitably qualified personnel to take radiographs. ${ }^{28}$

In conclusion, our methodology used computer software to detect and analyse the mandibular cortical width and when combined with clinical risk indices data detected patients at early, high risk of osteoporosis.
This work was supported by a research and technological development project grant from the European Commission Fifth Framework Programme 'Quality of Life and Management of Living Resources' (QLK6-2002-02243; 'OSTEODENT').

1. Dharmarajan TS, Banik P. Hip fracture. Risk factors, preoperative assessment, and postoperative management. Postgrad Med 2006; 119: 31-38.

2. Peng E W, Elnikety S, Hatrick N C. Preventing fragility hip fracture in high risk groups: an opportunity missed. Postgrad Med J 2006; 82: 528-531.

3. Ben Sedrine W, Broers P, Devogelaer J-P, Depresseux $\mathrm{G}$ et al. Interest of a prescreening questionnaire to reduce the cost of bone densitometry. Osteoporos Int 2002; 13: 434-442.

4. Pongchaiyakul C, Nguyen N D, Eisman J A, Nguyen T V. Clinical risk indices, prediction of osteoporosis, and prevention of fractures: diagnostic consequences and costs. Osteoporos Int 2005; 16: $1444-1450$.

5. Harrison E J, Adams J E. Application of a triage approach to peripheral bone densitometry reduces the requirement for central DXA but is not cost effective. Calcif Tissue Int 2006; 79: 199-206.

6. Devlin H, Allen P D, Graham J, Jacobs R et al. Automated osteoporosis risk assessment by dentists: a new pathway to diagnosis. Bone 2007; 40: 835-842.

7. Karayianni K, Horner K, Mitsea A, Bourkas L et al. Accuracy in osteoporosis diagnosis of a combination of mandibular cortical width measurement on dental panoramic radiographs and a clinical risk index (OSIRIS): the OSTEODENT project. Bone 2007; 40: 223-229.

8. Allen P D, Graham J, Farnell D J J, Harrison E J et al. Detecting reduced bone mineral density from dental radiographs using statistical shape models. IEEE Trans. IT in Biomed 2007; 11: 601-609

9. Cootes T F, Taylor C J, Cooper D H, Graham J. Active shape models - their training and application. Comput Vis Image Underst 1995; 61: 38-59.

10. Sedring W B, Chevallier T, Zegels B, Kvasz A et al. Development and assessment of the Osteoporosis Index of Risk (OSIRIS) to facilitate selection of women for bone densitometry. Gynecol Endocrinol 2002; 16: 245-250.

11. Richy F, Gourlay M, Ross P D, Sen S S et al. Validation and comparative evaluation of the osteoporosis self-assessment tool (OST) in a Caucasian population from Belgium. Q J Med 2004; 97: 39-46.

12. Devlin H, Horner K. Mandibular radiomorphometric indices in the diagnosis of reduced skeletal bone mineral density. Osteoporos Int 2002 13: 373-378.

13. Deeks J J. Systematic reviews of evaluations of diagnostic and screening tests. In Egger M, Smith
G D, Altman D G (eds). Systematic reviews in health care: meta-analysis in context, $2^{\text {nd }} \mathrm{ed} . \mathrm{pp}$ 255-256. London: BMJ Publishing Group, 2001.

14. Johansson $\mathrm{H}_{1}$ Oden $\mathrm{A}$, Johnell $\mathrm{O}$, Jonsson $\mathrm{B}$ et al. Optimization of BMD measurements to identify high risk groups for treatment - a test analysis. J Bone Miner Res 2004; 19: 906-913.

15. van Staa T P, Geusens P, Kanis J A, Leufkens H G $M$ et al. A simple clinical score for estimating the long-term risk of fracture in post-menopausal women. Q J Med 2006; 99: 673-682.

16. Leigh J P, Hubert H B, Romano P S. Lifestyle risk factors predict healthcare costs in an aging cohort. Am J Prev Med 2005; 29: 379-387.

17. Kayan K, De Takats D, Ashford R, Kanis J A, McCloskey $\mathrm{E} V$. Performance of clinical referral criteria for bone densitometry in patients under 65 years of age assessed by spine bone mineral density. J Postgrad Med 2003; 79: 581-584.

18. Brown J E. Advances in dental imaging. Primary Dent Care 2001; 8: 59-62.

19. Gibbs S J. Biological effects of radiation from dental radiography. Council on Dental Materials, Instruments, and Equipment. J Am Dent Assoc 1982; 105: 275-281.

20. Kanis J A, Johnell O. Requirements for DXA for the management of osteoporosis in Europe. Osteoporos Int 2005; 16: 229-238.

21. Johnell O, Kanis J A, Oden A, Johansson $\mathrm{H}$ et al. Predictive value of BMD for hip and other fractures. J Bone Miner Res 2005; 20: 1185-1194.

22. Kanis J A. Diagnosis of osteoporosis and assessment of fracture risk. Lancet 2002; 359: 1929-1936.

23. National Osteoporosis Society Position statement on the use of peripheral X-ray absorptiometry in the management of osteoporosis. Bath, England, 2004.

24. Majumdar S R, Kim N, Colman I, Chahal A M et al. Incidental vertebral fractures discovered with chest radiography in the emergency department: prevalence, recognition, and osteoporosis management in a cohort of elderly patients. Arch Intern Med 2005; 165: 905-909.

25. Devlin C V, Horner K, Devlin H. Variability in measurement of radiomorphometric indices by general dental practitioners. Dentomaxillofac Radio/ 2001; 30: 120-125.

26. Ballard P A, Purdie D W, Langton C M, Steel S A, Mussurakis S. Prevalence of osteoporosis and related risk factors in UK women in the seventh decade: osteoporosis case finding by clinical referral criteria or predictive model? Osteoporos Int 1998; 8: 535-539.

27. Kanis J A, Pitt FA. Epidemiology of osteoporosis. Bone 1992; 13 (Suppl 1): S7-S15.

28. Rushton V E, Horner K, Worthington H V. The quality of panoramic radiographs in a sample of general dental practices. BrDent J 1999; 186: 630-633. 